\title{
An Improved Firefly Algorithm and its application in Time-table Problems
}

\author{
Jianjun Zhang ${ }^{1, \mathrm{a}} \quad$ Yueguang $\mathrm{Li}^{2, \mathrm{~b}}$ \\ ${ }^{1}$ Gansu Normal University for Nationalities, Hezuo, gansu, 747000, \\ China \\ ${ }^{2}$ Gansu Normal University for Nationalities, Hezuo, gansu, 747000, \\ China \\ ${ }^{a}$ Yueguangli7@163.com, ${ }^{b}$ Yueguangli7@sohu.com
}

\begin{abstract}
In this paper, according to the characteristics of time-table problem. A Novel Firefly Algorithm was used to solve time-table problem, the algorithm was experimented and the experimental results show that the new algorithm to be successful in time-table problem. The experimental result demonstrates that the Improve firefly algorithm can get better solutions to time-table problem
\end{abstract}

Keywords: Firefly Algorithm; Intelligent algorithm; Time-table problem

\section{Introduction}

The intelligent algorithm has been paid more and more attention, such as neural network, genetic algorithm, ant colony algorithm, particle swarm algorithm, which they are used to solve combinatorial optimization and $\mathrm{NP}^{[1]}$.

Time-table Problem of Universities is a many factor of the global optimization problem, it is a NP- complete problem ${ }^{[2]}$. Since Time-table Problem of Universities involved in many information, generally use the approximate optimal algorithms, such as genetic algorithm, simulated annealing algorithm ${ }^{[3]}$.

The firefly algorithm is the development of simulating biological characteristics of the adults firefly, it is a stochastic optimization algorithm, the current literature is two versions: a)It is proposed by India scholar Krishnanand ${ }^{[4]}$, the algorithm is called GSO (glowworm swarm optimization); b) It is proposed by Cambridge scholar Yang ${ }^{[5]}$, the algorithm is called FA (Firefly algorithm), the bionic principle of two kinds of algorithms is the same, but there are some differences in the specific implementation, this paper analyzes the bionics principle of firefly algorithm, and definite the optimization process of the algorithm from the angle of mathematics, through testing Time-table Problem of Universities to verify the feasibility and effectiveness of the algorithm.

\section{The basic principle of the firefly algorithm}




\subsection{Mathematical description and analysis of the algorithm}

The firefly algorithm includes two factors: the brightness and the degree of attraction. The brightness of the firefly reflects the pros and cons of location and determines its direction of movement, attraction degree determines the distance of the firefly mobile, constantly update the brightness and the attraction degree, so as to realize the goal of optimization. From a mathematical perspective describes the Firefly algorithm optimization mechanism, the description as follows ${ }^{[6,7]}$

(1) The relative fluorescence intensity of fireflies as:

$$
h=h_{0} /\left(1+z r_{i j}^{2}\right)
$$

Among them: ${ }^{h_{0}}$ for the maximum fluorescence intensity of fireflies, that is itself $(r=0)$ fluorescence brightness, associated with the value of the objective function, the objective function value is better, its brightness is higher; $z$ as the light absorption coefficient, because the fluorescence decreases with the increasing of distance and media absorption ${ }^{[8]}$, so set the light absorption coefficient to reflect this characteristic, it can set to constant; $r_{i j}$ is the space distance of the firefly $i$ and the firefly $j$.

(2) The firefly attraction degree is:

$$
\rho=\rho_{0} * e^{-z r_{i j}^{2}}
$$

Among them: $\rho_{0}$ as the biggest attraction, namely the light source $(r=0)$ attraction degree; $z, \quad r_{i j}$ meaning as above.

(3) The firefly $i$ is attracted to move to the firefly $j$, the location update by formula (3) decision.

$$
x_{i}^{\prime}=x_{i}+\rho^{*}\left(x_{j}-x_{i}\right)+\alpha *(\text { rand }-1 / 2)
$$

Among them: $x_{i}^{\prime}$ is the individual i towards a brighter individual j update location, $x_{i}, x_{j}$ are the firefly $i$ and the firefly $j$ located before the entire population renewal; $\alpha$ is the step factor, it is constant between 0 and 1 , rand is a random factor between 0 and 1,it obeys uniform distribution.

At present, it has been found that many insects existing Levy flight ${ }^{[9]}$, and Levy flight has been used in the field of optimization, and achieve the expected effect. In order to enhance the algorithm global search performance, avoid the population into a local optimum in the search process, if the individual is no better than their individual, choose to Levy flight instead of random flight in the 
original algorithm. In addition, the non - optimal those individuals in a population, the flight formula was improved: when they find more bright than their individual, first generates a random number $\mathrm{q}$ by the system, if $\mathrm{q}$ is less than 0.5 , the formula (4) is updated; otherwise, still use the formula (3) to update the individual position.

$$
x_{i}^{\prime \prime}=x_{i}^{\prime}+\rho^{*}\left(x_{j}-x_{i}^{\prime}\right)+\alpha *(\text { rand }-1 / 2)
$$

Among them,: ${ }^{x_{j}}$ still expresses renewal position of individual $j$ before the entire population, $x_{i}^{\prime}$ expresses the individual i toward the front of $j-1$ individuals than their bright individual after the update new position, $x_{i}$ expresses $x_{i}^{\prime}$ toward than their bright individual $j$ after update location, $\rho$ expresses that individual $j$ appeal to the individual $i$. As can be seen, formula (4) is updated in real time, formula (3) depends only on the entire population before moving. This flight update can increase the randomness of flight, It is helpful to keep the diversity of population, increasing the population search space.

In order to accelerate the convergence of the population, the paper proposes a method of $\alpha$ updating, which $\alpha$ gradually decreases with increasing number of iterations. Update formula is as follows:

$$
\alpha=\alpha_{0}-e^{-0.001^{*} t}
$$

In the formula (5): $\alpha_{0}$ is $0.9, \mathrm{t}$ is number of iterations.

\section{Improved firefly algorithm}

The concrete steps of the algorithm are as follows:

1) Initialization the basic parameter of algorithm. Set the number of fireflies is $s$, the biggest attraction is $\rho_{0}$, light absorption coefficient is $z$, the random parameters is $\alpha_{0}$, the maximum number of iterations is $t_{\max }=1000$, the evolving algebra $t=0$.

2) Randomly initialization firefly position, calculate the objective function value of firefly as the respective maximum fluorescence intensity of $h_{0}$.

3) By formula 1 and formula 2 to calculate the relative brightness of $h$ and attraction $\rho$, according to relative brightness of $h$ to decide the movement direction of the firefly.

4) The formula 5 is used to update $\alpha$, the individual, if there is more lighter than its individual, in accordance with the update is improved, by formula (4) to update the individual position; otherwise, the Levy flight is used to update the individual location. 
5) According to the updated firefly position, to recalculate brightness of the firefly.

6) The system generates a random number, if the random number is less than the local search probability $p$, then local search of individuals of the population, and regenerate population.

7) Determine whether meet the conditions of termination of the algorithm. Such as the maximum number of iterations $t_{\text {max }}$ is1000 or best solution stagnation does not change, turn to step 7 , or $t=t+1$, turn to step 3 .

8) Output global extreme value point and optimal individual value.

\section{The test results}

The following, Department of Computer Science of Gansu Normal College for Nationalities (4grades, 72 classes, 400 teachers) as an example to test, and record test results, the actual inspection generation schedule, no case of conflict. In the figure 1, it shows that the average case with iteration times of the improved firefly algorithm and the firefly algorithm in the search for the optimal value. From the figure, we can see that the steps of the improved firefly algorithm for each iteration requires the operation has increased, but the convergence of the improved firefly algorithm is significantly less than the firefly algorithm, but also it improves the average search results. Table 1 gives that the data of the improvement firefly algorithm, particle swarm algorithm, genetic algorithm solving university time table problem of the optimal value, average distance, relative error and average running time. Experimental results show that, the improved firefly algorithm compared with other two kinds of optimization algorithms in solving the problem of university time table, the more stable performance. 


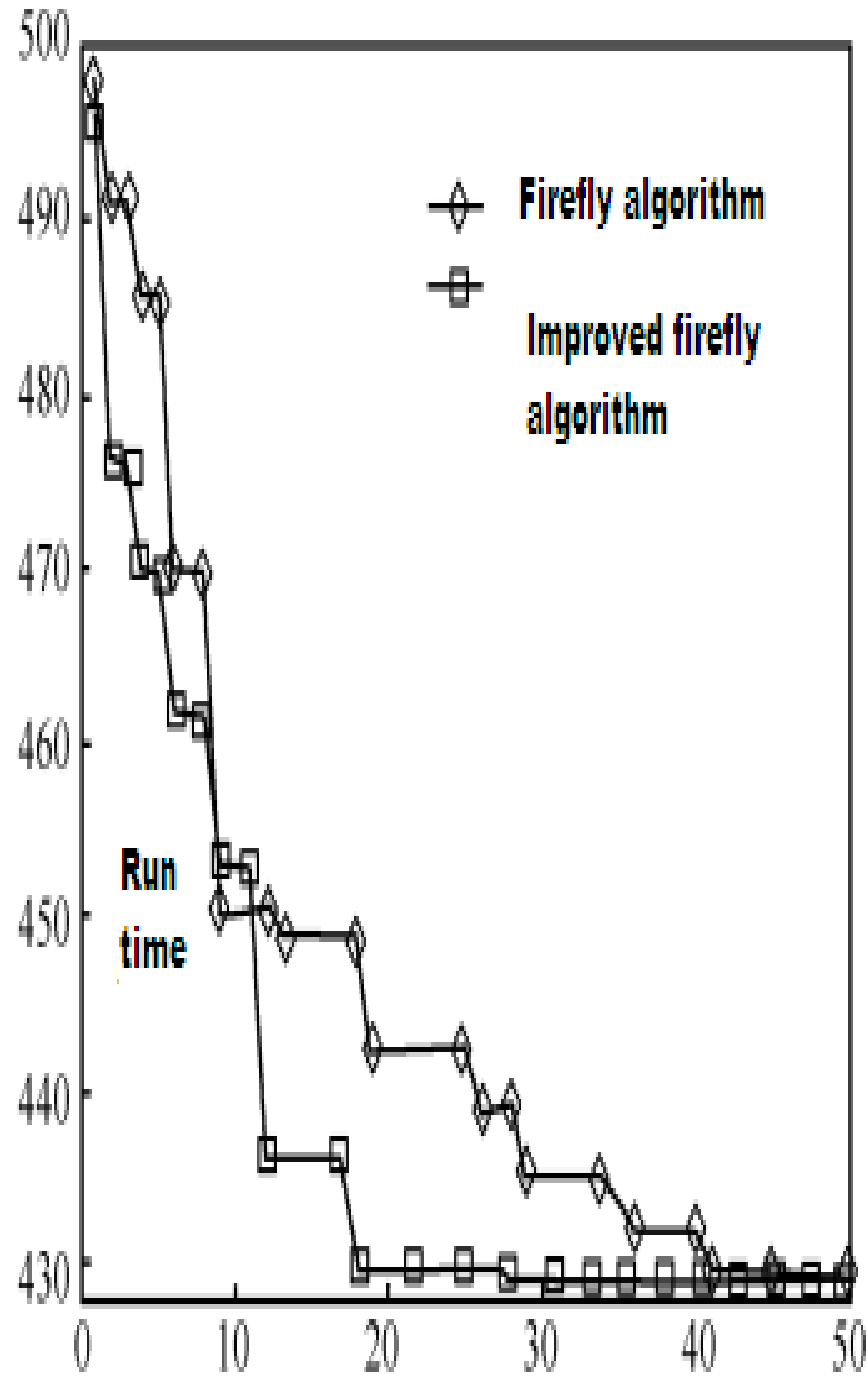

The number of iterations

Figure 1 the compared of search convergence speed of firefly algorithm and the improved firefly algorithm to solve university timetable problem

Table 1 the Comparison of the algorithm of the paper, particle swarm algorithm and genetic algorithm 


\begin{tabular}{|c|c|c|c|c|}
\hline The algorithm & $\begin{array}{c}\text { The average } \\
\text { distance }\end{array}$ & $\begin{array}{c}\text { the } \\
\text { optimal value }\end{array}$ & $\begin{array}{c}\text { relative } \\
\text { error } \\
(\%)\end{array}$ & $\begin{array}{c}\text { average } \\
\text { running time } \\
(\mathrm{s})\end{array}$ \\
\hline $\begin{array}{c}\text { the algorithm } \\
\text { of the paper }\end{array}$ & 430.54 & 428.56 & 0.39 & 23.16 \\
\hline $\begin{array}{c}\text { particle } \\
\text { swarm } \\
\text { algorithm }\end{array}$ & 440.78 & 436.77 & 2.77 & ----- \\
\hline $\begin{array}{c}\text { genetic } \\
\text { algorithm }\end{array}$ & 437.83 & 431.99 & 2.09 & 18.44 \\
\hline
\end{tabular}

\section{Conclusions}

The firefly algorithm is a new evolutionary algorithm, although the study is not long, the lack of solid theoretical foundation, but it has strong robustness and parallel computing, etc. This paper proposes an improve firefly algorithm. The improve firefly algorithm is applied to solve university timetable problem, the performance has been improved obviously. Experiments show that: the algorithm is feasible and effective for solving university timetable problem.

\section{References}

[1] Reingold. E. M. J. Neivergelt and N. Deo. Combinatorial Algorithms: Theory and practice Prentice-Hall ,Englewood cliffs, NJ(1977).

[2] Garey M R, Johnson D S. Computers and Intractability: A Guide to the Theory of NP-Completeness[M]. San Franciso, CA: Freeman,4,109-122(1979).

[3] Ibrahim H Osman. Heuristics for the Generalised Assignment Problem: Simulated Annealing and Tabu Search Approaches OR Specktrum, 17,211-255(1995).

[4] Krishnanand K N, Ghose D. Detection of multiple source locations using a glowworm metaphor with applications to collective robotics [C] / /Proc of IEEE Swarm Intelligence Symposium. Piscataway: IEEE Press, 2005: 84-91.

[5] Yang Xinshe. Nature inspired meta heuristic algorithms [M] . [S. 1. ] : Luniver Press, 2008: 83-96.

[6] Yang Xinshe. Firefly algorithms for multimodal optimization [C] / /Proc of the 5th International Symposium on Stochastic Algorithms: Foundations and Applications. 2009: 169-178. 
[7] Yang Xinshe, DEB S. Eagle strategy using lévy walk and firefly algorithms for stochasticoptimization $[\mathrm{J}]$. Studies in Computational Intelligence, 2010, 284: 101-111.

[8] Yang Xinshe. Firefly algorithms for multimodal optimization[C]//Proceedings of the 5th International Conference on Stochastic Algorithms: Foundations and Applications. Berlin/Heidelberg, Germany: Springer-Verlag, 2009: 169-178.

[9] Brown C T, Liebovitch L S, Glendon R. Lévy flights in Dobe Ju' hoansi foraging patterns[J]. Human Ecology, 2007, 35(1): 129-138. 